\title{
Modified Natural Rubber Induced Aqueous to Toluene Phase Transfer of Gold and Platinum Colloids
}

\author{
Mohamad Abu Bakar, Jamil Ismail, Cheng Hoon Teoh, Wei Leng Tan, and Noor Hana Hanif Abu Bakar \\ Nanoscience Research Laboratory, School of Chemical Sciences, University of Sains Malaysia, Penang 11800, Malaysia
}

Correspondence should be addressed to Mohamad Abu Bakar, bmohamad@usm.my

Received 9 November 2007; Accepted 7 January 2008

Recommended by Zhili Xiao

Citrate-stabilized gold and platinum particles were prepared in aqueous phase and transferred to toluene phase by employing 2-propanol as the transfer agent. It was found that the modified natural rubber (ENR) induced the phase transfer and assisted the dispersion of the citrate-free metal particles into the organic phase. The amounts of gold and platinum transferred are $93.4 \%$ and $86.1 \%$, respectively. This phase transfer technique produced organosols of smaller particle sizes and narrower size distribution with self-assembly arrangements when compared to those prepared via the previous in situ preparations. The respective average particle size and standard deviation of gold before and after phase transfer were $6.3 \pm 1.7 \mathrm{~nm}$ and $7.2 \pm 1.3 \mathrm{~nm}$, while for platinum they were $4.0 \pm 0.7 \mathrm{~nm}$ and $4.2 \pm 0.8 \mathrm{~nm}$. The slight increase in the average sizes and overall size distributions in both metals after transfer was attributed to multiparticle aggregation in the organic phase.

Copyright (c) 2008 Mohamad Abu Bakar et al. This is an open access article distributed under the Creative Commons Attribution License, which permits unrestricted use, distribution, and reproduction in any medium, provided the original work is properly cited.

\section{INTRODUCTION}

Methods for the preparation of nanosized noble metals have been widely explored due to their importance in various applications [1]. Among the applications are sensors [2], catalysts [3], labels [4], as well as optical [5] and electronic materials [6]. In order to meet the requirements of these applications, particle synthesis has been tailored to meet specific features such as dispersity, size, and shapes [7]. These properties are dependent on the preparation environment and techniques. There are various routes to synthesize metal particles. Reduction methods such as chemical [8], sonochemical [9], electromagnetic [10], and electrochemical [11] have been reported. Chemical reduction is the most favored as a "bottomup" approach.

It is well known that metal particles can be easily formed in aqueous compared to organic phase due to the ease of solubility of most metal precursors, chemical reducing agents (e.g., borohydrides and citrates), and stabilizers in water. This has afforded a wide range of metal hydrosols with various particle sizes and morphology as compared to metal sols prepared in the organic environment. To diversify the range of metal organosols, phase transfer of metal particles from aqueous to organic phase was introduced. In general, phase transfer technique has many advantages due to a number of factors. Among them is the ease of particles in the organic phase to adopt the size, size distribution and morphology of the particles prepared in aqueous phase. In addition, the particle size and morphology can be better manipulated due to the hydrosols that are already well formed prior to the particles transfer to the organic phase. The interaction between the metals and the organic solvents with or without stabilizer often creates particles self-assembly and this is advantageous in certain applications. Furthermore, aqueous to organic phase method enables the use of nonwater soluble stabilizers/matrix.

Transfer agents and particle stabilizers are often needed for such particle phase transfer processes. Among the transfer agents commonly used are tetraoctylammonium bromide (TOAB) [12], dimethyldioctadecylammonium chloride (DDAC) [13], concentrated hydrochloric acid [14], and alcohols (e.g., ethanol and 2-propanol) [15]. The common particle stabilizers are alkylamines (e.g., octadecylamine and dodecylamine) [16], alkylthiols (1-dodecanethiol) [17], citrates, and surfactants (e.g., mercaptopropionic acid) [18]. A few of these stabilizers are also known to act as transfer 
agents $[16,17]$. Few polymers have been employed as stabilizers for particles in the organic phase. Esumi et al. employed a synthetic poly (amidoamine) dendrimer to encapsulate gold particles in an organic phase [19]. There are also few reports on the usage of natural polymers as stabilizers in a similar phase transfer process. We have previously reported the use of modified natural rubber (ENR-50) as the stabilizing agent or matrix for the silver nanoparticles in the organic phase [20]. Our work uses surfactant, tetraoctylammoniumbromide (TOAB), as the transferring agent. However, a trace presence of TOAB in the organic phase was observed. Therefore, the matrix comprising a mixture of stabilizer (TAOB) and polymer (ENR) encapsulates the metal particles. This can affect the properties of the resultant composite especially in certain applications such as in electrical conductive adhesive (ECA), thermal interface material (TIM), and polyelectrolyte. For the said applications, bare metal-polymer composites are much desired. The use of citrate as aqueous phase particle stabilizer is of particular interest as compared to alkylamines and alkylthiols. Unlike the latter, citrate has been reported to be completely detached or replaced by other organic soluble stabilizers upon particle transfer into the organic phase [16-18].Thus, in this study, we report the use of ENR-50 that induces the aqueous to toluene transfer of citrate-stabilized gold and platinum particles and the ability of the ENR-50 to exert good dispersion of the citrate-free particles in the toluene layer.

\section{EXPERIMENTAL}

\subsection{Materials}

Hydrogen tetrachloroaurate trihydrate, $\mathrm{HAuCl}_{4} \cdot 3 \mathrm{H}_{2} \mathrm{O}$ and platinic chloride, $\mathrm{H}_{2} \mathrm{PtCl}_{6}$ (both from Sigma, USA), sodium borohydride, $\mathrm{NaBH}_{4}$ (Riedel-de Haen, Germany), trisodium citrate, $\mathrm{Na}_{3} \mathrm{C}_{6} \mathrm{H}_{5} \mathrm{O}_{4} \cdot 2 \mathrm{H}_{2} \mathrm{O}$ (Ajax Chemicals, Australia), toluene (Fisher Chemicals, UK), and 2-propanol (Merck, Germany) were obtained commercially and used without further purification. Modified natural rubber with 50\% epoxidation (ENR-50) was supplied by Guthrie Polymer Sdn. Bhd (Malaysia) and purified according to the previous report [21]. The average molecular weight of the purified ENR-50 is 400000 .

\subsection{Preparation and phase transfer of metal colloids}

\subsubsection{Metal colloids}

For the preparation of $20 \mathrm{~mL}$ aqueous gold colloids, $1.56 \mathrm{~mL}$ $2.54 \times 10^{-6} \mathrm{~mol} \mathrm{~mL}^{-1}$ of aqueous $\mathrm{HAuCl}_{4} \cdot 3 \mathrm{H}_{2} \mathrm{O}$ stock solution was pipette into a reaction flask and $18.04 \mathrm{~mL}$ of distilled water was added. The solution mixture was stirred with magnetic stirrer. A $0.4 \mathrm{~mL}$ of $3.0 \times 10^{-5} \mathrm{~mol} \mathrm{~mL} \mathrm{~mL}^{-1}$ trisodium citrate solution was then added and stirring was continued for a further 10 minutes. Subsequently, $0.4 \mathrm{~mL}$ of $1.0 \times$ $10^{-4}$ mol mL ${ }^{-1}$ of freshly prepared, chilled $\mathrm{NaBH}_{4}$ solution was added.

Similarly, for the preparation of $20 \mathrm{~mL}$ aqueous platinum colloids, $1.44 \mathrm{ml}$ of $2.74 \times 10^{-6} \mathrm{~mol} \mathrm{~mL}^{-1}$ aqueous $\mathrm{H}_{2} \mathrm{PtCl}_{6}$ stock solution and $18.16 \mathrm{~mL}$ of distilled water was mixed and stirred. A $0.4 \mathrm{~mL}$ of $3.0 \times 10^{-5} \mathrm{~mol} \mathrm{~mL}^{-1}$ solution of trisodium citrate was then added and stirred for another 10 minutes. This was followed by the addition of $2.5 \mathrm{~mL}$ of $1.0 \times 10^{-4} \mathrm{~mol} \mathrm{~mL}^{-1}$ of chilled $\mathrm{NaBH}_{4}$ solution.

\subsubsection{Phase transfer}

A $5 \mathrm{~mL}$ of the respective metal colloids prepared above was added into a flask containing $5 \mathrm{~mL}$ of 2-propanol followed by vigorous stirring. A $5 \mathrm{~mL}$ of $2.50 \times 10^{-5} \mathrm{~mol} \mathrm{~mL}^{-1}$ of ENR-toluene stock solution was then added. The stirring was stopped after 1 minute and the mixture was left standing for phase separation.

The role of 2-propanol and ENR in the phase transfer process was also investigated. Thus, experiments without the 2-propanol as phase transfer agent and another in the absence of ENR in the organic phase were similarly carried out as above.

\subsection{Characterizations}

Prior to any form of characterization, the organic layer of each preparation was separated and evaporated off. After that, $5 \mathrm{~mL}$ of toluene was added to redisperse the metal colloids and these were subsequently used for various characterizations as described below.

The size and morphology of the metal particles before (aqueous phase) and after (organic phase) phase transfer was analyzed using a Philip CM12 transmission electron microscope (TEM) operating at $80 \mathrm{kV} .5$ drops of the respective metal colloids were cast onto a carbon coated copper grids and left to dry. The average size of the particles, standard deviations (SD), and size distributions were evaluated using the computer software "Analysis Docu 2.11" (Soft Imaging System GmbH, Munster, Germany). The optical property of the metal colloid was measured using a Hitachi U-2000 UV-vis spectrophotometer. The metal colloid was placed in a $1 \mathrm{~cm}$ quartz cuvette and measured within the range of 200$800 \mathrm{~nm}$. X-ray diffraction analysis was performed using a Siemens D5000 X-ray diffractometer with a monochromatic $\mathrm{Cu}-\mathrm{K} \alpha$ radiation filter in the $2 \theta$ range of $0-100^{\circ}$. Samples were prepared as a thin film on a glass slide. The metal contents of the organic phase after phase transfer were determined using the Perkin Elmer 3100 flame atomic absorption spectrometer (AAS). The average molecular weight of purified ENR was determined using a Perkin Elmer 250 gas permeation chromatography (GPC). The structure of ENR was analyzed using Perkin Elmer System 2000 FT-infrared spectrometry.

\section{RESULTS AND DISCUSSION}

\subsection{Synthesis}

Aqueous solutions of chloroauric acid and chloroplatinic acid in the presence of trisodium citrate were yellow and pale 


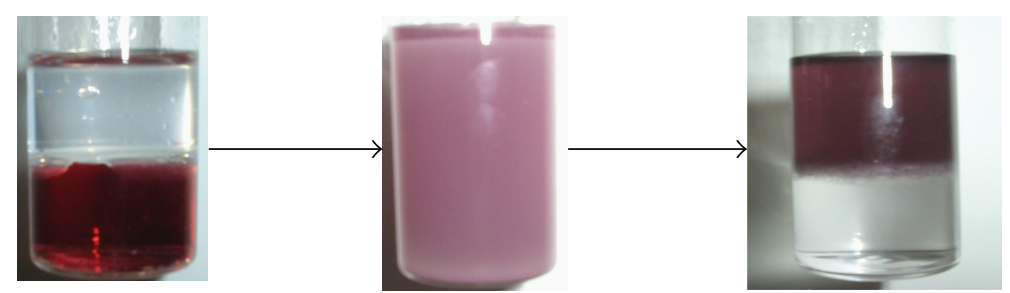

(i)

(ii)

(iii)

(a)

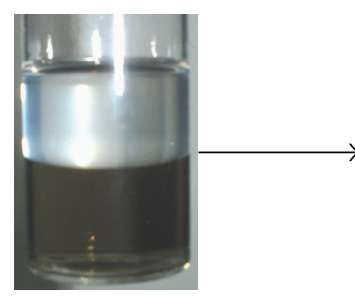

(i)

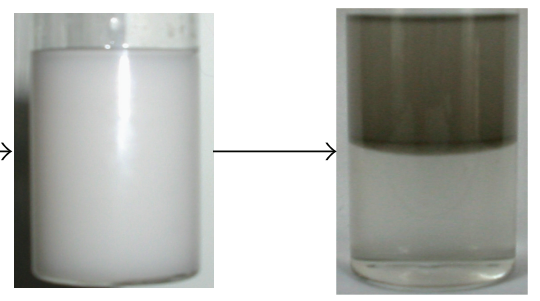

(ii) (iii)

(b)

Plate 1: (a) Gold nanoparticles and (b) platinum nanoparticles at various stages of phase transfer: (i) before mixing, (ii) during mixing, and (iii) after phase separation.

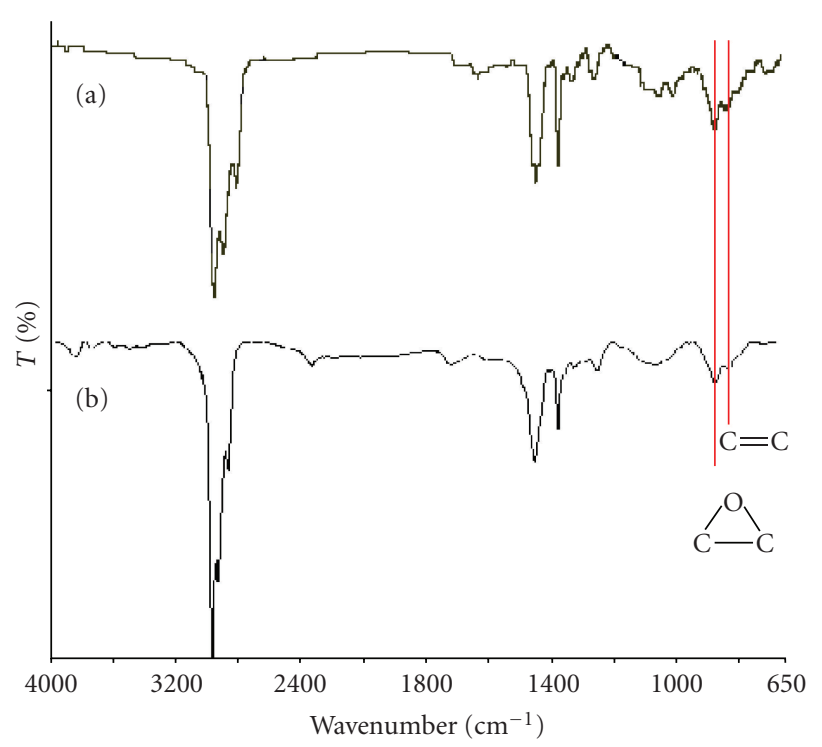

FIgURE 1: Typical FTIR spectra for the metal-ENR-50 organosol.

yellow, respectively. Upon addition of sodium borohydride to the aqueous gold solution, the reduction process causes the color to progressively change from dark blue to purple then pink and finally wine-red which are the typical colors for citrate-stabilized metallic goldnanoparticles [22]. For the citrate-stabilized platinum nanoparticles, the color however changed from light brown to dark brown [15]. Equations
(1) represent borohydride reductions of the respective metals [23]:

$$
\begin{aligned}
\mathrm{NaBH}_{4} & +\mathrm{HAuCl}_{4}+3 \mathrm{H}_{2} \mathrm{O} \\
& \longrightarrow \mathrm{Au}^{0}+\mathrm{H}_{3} \mathrm{BO}_{3}+3 \mathrm{HCl}+\mathrm{NaCl}+\frac{5}{2} \mathrm{H}_{2} \\
\mathrm{NaBH}_{4} & +\mathrm{H}_{2} \mathrm{PtCl}_{6}+3 \mathrm{H}_{2} \mathrm{O} \\
& \longrightarrow \mathrm{Pt}^{0}+\mathrm{H}_{3} \mathrm{BO}_{3}+5 \mathrm{HCl}+\mathrm{NaCl}+2 \mathrm{H}_{2} .
\end{aligned}
$$

When the respective citrate-stabilized metal hydrosol was stirred in the presence of ENR-toluene solution and 2propanol, a milky emulsion was formed. The emulsion for gold was milky pink while that of platinum was milky white as shown in Plates 1(a)(ii) and 1(b)(ii), respectively. After phase separation, the bottom aqueous layer becomes colorless while the top organic layer adopts the color of the respective metal hydrosols, (Plates 1(a)(iii) and 1(b)(iii)). However, when 2-propanol was excluded from the preparation, no apparent transfer of the particles between the phases was observed, as indicated by the color of the respective phases. They retained their original colors. There was no exchange of particles between the phases although contact between the aqueous and organic interface was intentionally increased by stirring. Therefore, the particles phase transfer was assisted by 2-propanol which acted as the transfer agent. 2-propanol has a mutual solubility in aqueous and toluene solvents. Alcohol is known to act both as solvent and reducing agent for noble metals. However, the latter is only possible at elevated temperatures. The work described uses 2-propanol solely as the phase transfer agent that acts as the intermediary for both phases thus enabling the transfer of the metal particles between phases. 


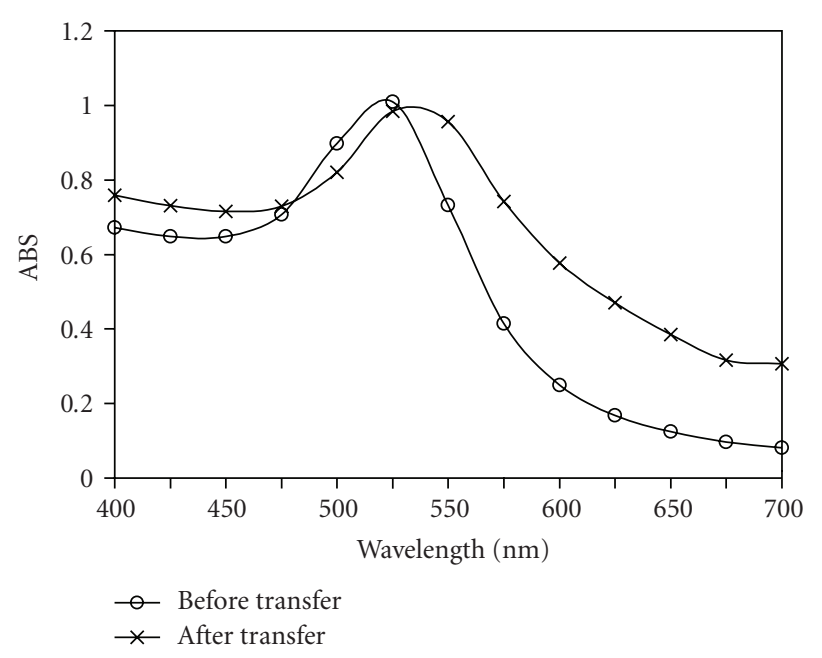

Figure 2: UV-Vis absorbencies of gold sols.

For these metal organosols, there are no significant changes observed from the FTIR spectra of ENR before and after phase transfer. A typical IR spectrum of the metalENR-50 organosol is presented in Figure 2. The peaks due to the oxirane at $877 \mathrm{~cm}^{-1}$ [24] and the $\mathrm{C}=\mathrm{C}$ at $840 \mathrm{~cm}^{-1}$ remained unchanged. Therefore, it is anticipated that there are no structural changes of the ENR chain. There was also no FTIR band attributable to the citrate observed in the organic phase, which meant that citrate was not transferred into the toluene phase. This implied that citrate stabilizers remained in the aqueous phase (probably due to the insolubility of citrate in toluene) after the transfer process of the respective metal particles to the organic phase.

The optical properties of the gold sols before (hydrosol) and after (organosol) phase transfer are shown in Figure 3. The citrate-stabilized gold hydrosol showed a typical absorbance at $\lambda_{\max } 525 \mathrm{~nm}$ that corresponds to the surface plasmon resonance (SPR) of gold nanoparticles [25]. After phase transfer, the $\lambda_{\max }$ is slightly red shifted to $530 \mathrm{~nm}$. This red shift often indicated the formation of bigger particles but in this work, it may also be due to the variation in the solvent's refractive index on going from aqueous to toluene [16]. Other possible factors that may have caused this red shift are the changes in the adsorbed molecules on the particle surface (i.e., citrate stabilized to ENR stabilized) or the aggregation of the particles during the transfer process as previously reported [15]. Platinum sols do not exhibit SPR within the UV-Vis range studied.

The organic layers after phase transfer were subjected to the atomic absorption spectrophotometry (AAS). The AAS analyses of the organic aliquots showed that the average metal contents in the organic phase after the transfer of the metal particles are $93.4 \%$ and $86.1 \%$ for gold and platinum, respectively.

In order to ascertain the role of ENR as the stabilizer, an experiment was carried out using only citrate-stabilized metal hydrosols that were transferred into toluene without ENR. It was found that the particles tend to aggregate forming several masses of particles at the interphase as shown in
Plate 4. This observation indirectly proved that the transportation of citrate into the organic phase did not occur. This is due to the low solubility of citrate in the toluene. Thus, without any stabilizer in the organic phase, the metal particles could not disperse well and therefore tend to agglomerate. Therefore, in those experiments where the ENR is included in the organic phase, the ENR is responsible for dispersing and stabilizing the transferred metal particles in the organosols. The ability of ENR to act as a stabilizer arises from its molecular structure and its chain conformation when in toluene solution. The degree of epoxidation, distribution of the epoxide group in the polymer chain, molecular weight and interaction between the chain and toluene molecules determined the chain conformation. In the case of ENR-50, an isoprene polymer with 50\% mol epoxidation level and the average molecular weight of 400,000 , the polymer chain resumes an extended coil conformation. This conformation is capable of accommodating the metal particles, brought to proximity by the carrier 2-propanol, and results in the metal particles entrapment in the coiled chains. This mode of particle stabilization is generally termed as trapped within the voids of the polymer chains [20]. Thus, the particle aggregations are inhibited. Therefore, in the presence of ENR, the ENR induces the transfer of metal particles (into the organic phase), assists in the particle dispersion by providing sites (voids) for the particle, and stabilizes the particles (within the voids) in the organosols. Although it has been reported that metal particles can interact with epoxide and the $\mathrm{C}=\mathrm{C}$ groups of the ENR to form metal-complexes $[26,27]$, there is no evidence of such interactions or complexations was observed from the FTIR spectra as mentioned earlier.

\subsection{Phase and morphology of gold and platinum particles}

The ENR-gold organosol cast into film exhibited diffraction peaks at $2 \theta$ of $38.2^{\circ}, 44.4^{\circ}, 64.6^{\circ}$, and $77.6^{\circ}$ that corresponded to the lattice planes of [111], [200], [220], and [311], respectively, Figure 5(a). Similarly, the ENR-platinum film also exhibited the diffraction peaks corresponding to the lattice planes of [111], [200], [220], and [311] at the respective $2 \theta$ of $39.7^{\circ}, 46.2^{\circ}, 67.4^{\circ}$, and $81.2^{\circ}$, Figure $5(\mathrm{~b})$. These lattice planes corresponded to the face-centered cubic (fcc) lattice of metallic gold and platinum, respectively $[28,29]$. The very broad peak at $2 \theta$ range of $\sim 10-30^{\circ}$ in both diffractograms was due to the halo-ENR.

The TEM micrographs and histograms for gold and platinum particles before and after phase transfer are depicted in Figure 6. The gold and platinum particles were selfassembled. The shapes of the particles in the aqueous phase are similar as those described by Yang et al. [15] using citrate as the stabilizer. The shape is basically retained upon the transfer to the organic phase. However, the platinum particles are less defined as compared to gold probably due to its smaller size. The average sizes and standard deviations of the gold particles before and after phase transfer are $6.3 \pm 1.7 \mathrm{~nm}$ and $7.2 \pm 1.3 \mathrm{~nm}$, respectively. For platinum particles, however, the respective average sizes and standard deviations before and after phase transfer are $4.0 \pm 0.7 \mathrm{~nm}$ and $4.2 \pm 0.8 \mathrm{~nm}$. 


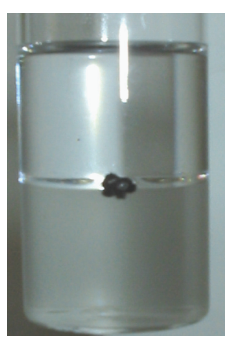

(a)

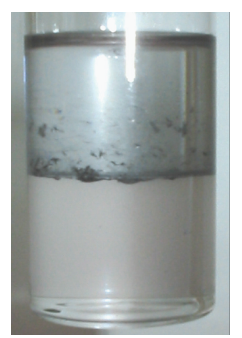

(b)

Plate 2: Phase transfer in the absence of ENR for (a) gold and (b) platinum.

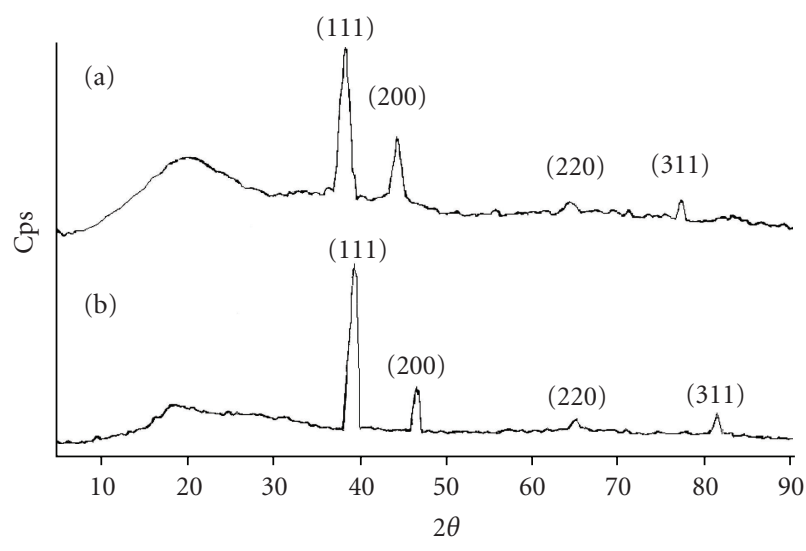

FIGURE 3: XRD diffractograms of cast ENR-stabilized metal particles film of (a) gold and (b) platinum.

Generally, there is a slight increase in particle size and size distribution after phase transfer. For gold, the majority of the particles are situated around 6-7 nm range before the phase transfer. However, the amount of particles in the size range of 7-8 $\mathrm{nm}$ increased after phase transfer. Similarly, for platinum, the increase is from around 3-4 $\mathrm{nm}$ to $5-6 \mathrm{~nm}$ size range. Especially for gold, the increases in particle sizes and size distributions are in accordance with the red shift of the $\lambda_{\max }$ as observed in the UV-Vis analysis. Generally, the increase in particle size may be due to the slight aggregation of the particles upon transfer from the aqueous to the organic phase [15]. As the particles move into the organic phase, they may come in contact with each other and aggregate due to the absence of citrate surrounding the particles before the particles are finally surrounded by ENR and hence inhibiting further growth.

\subsection{Mechanism of interphase particles transfer}

In the various phase transfer experiments, it was found that very little transfer of particles was observed without stirring. Similarly, without 2-propanol there is also no apparent particle transfer observed, even after prolonged stirring. In the absence of ENR, the metal particles tend to aggregate and accumulate at the aqueous/toluene interphase. For those successful phase transfer processes, no citrate was detected in the organic phase. This inferred that the metal particles un- derwent an exchange of stabilizers upon phase transfer, that is, from citrate stabilized in aqueous phase to ENR stabilized in the toluene phase. Therefore, addition of 2-propanol is required for phase transfer to occur with 2-propanol acting as the transfer agent while ENR acts as replacement stabilizer and therefore exerts dispersion of the particles in the toluene phase. It must be noted that in the several mechanisms previously reported, the stabilizers are often transferred along with the metal particles from the aqueous to organic phase, that is, without undergoing any exchange of stabilizers [3033].

In this work, the probable mechanism of phase transfer is proposed as follows. Upon formation of the metal particles, they are initially stabilized by the citrate in the aqueous phase. The microemulsion was formed when hydrosol and ENR toluene in the presence of 2-propanol were mixed. The formation of the microemulsion increases the surface area between the organic and aqueous phase. The addition of 2propanol will cause the transfer of the citrate-stabilized particles into the toluene layer due to the mutual solubility of alcohol in both solvents. Upon particle transfer, the particles undergo replacement of stabilizer from the citrate to ENR. This is facilitated by the structure of ENR-50 that is naturally loose and contains many interchain voids [34]. The citrate disentangled from the surface of the metal particles and remained in the aqueous layer as it is insoluble in toluene as previously evidenced by the FTIR results. Therefore, the presence of the ENR in the organic phase induces dispersion of the metal particles. The metal particles diffused into the voids and formed stable ENR-metal organosols as discussed earlier.

\section{CONCLUSION}

Citrate-stabilized gold and platinum particles had been prepared in aqueous phase via sodium borohydride reduction of their respective salts and subsequently transferred to the organic phase using 2-propanol as the transfer agent. An absence of ENR in the organic layer caused the metal particles to accumulate and form aggregates at the interphase. The presence of ENR in the organic phase induced the metal particles dispersity with only a minimal growth of the metals particles after phase transfer. Therefore, ENR can be used as a matrix and/or stabilizer for the transfer of citratestabilized gold and platinum hydrosol to the organosol. The dried metal colloids can be redispersed using various 

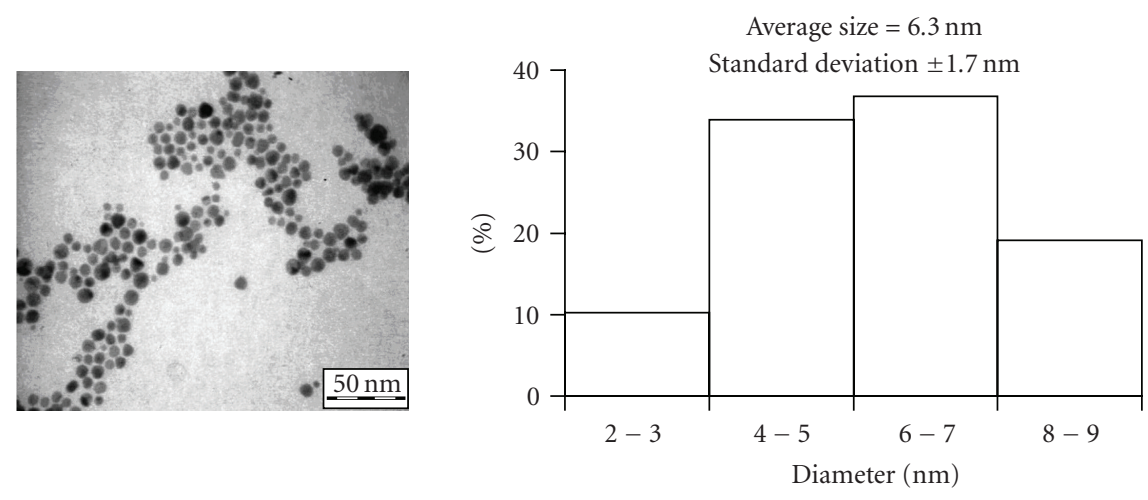

(a)
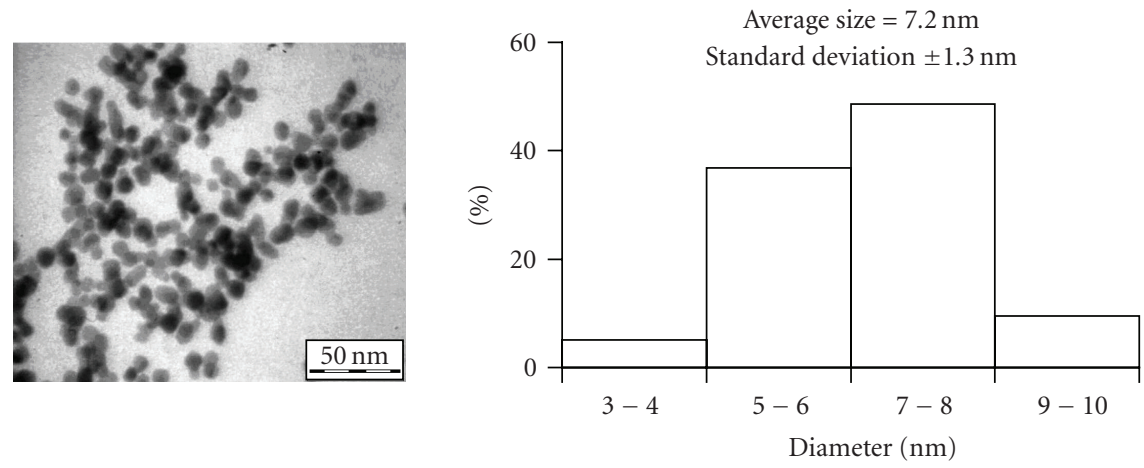

(b)
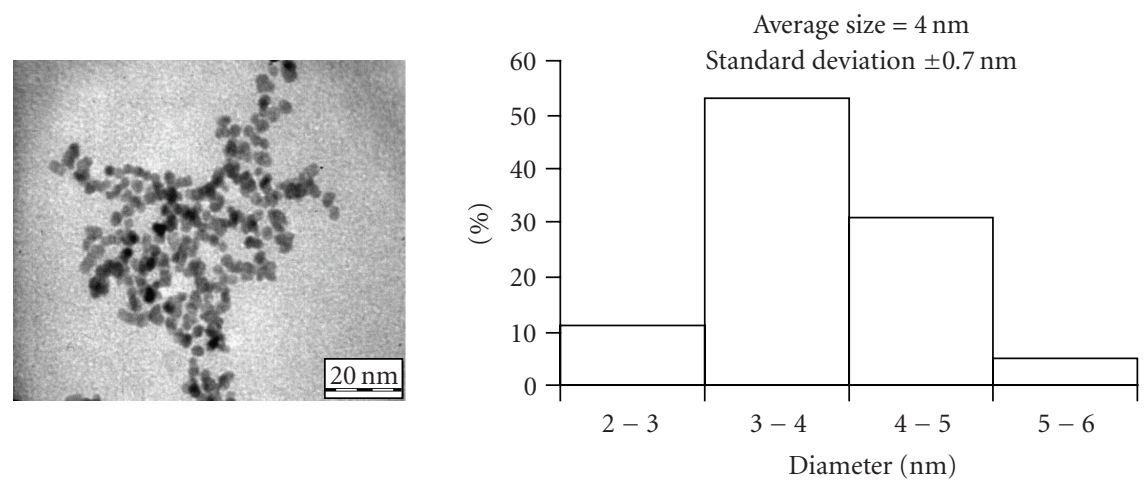

(c)
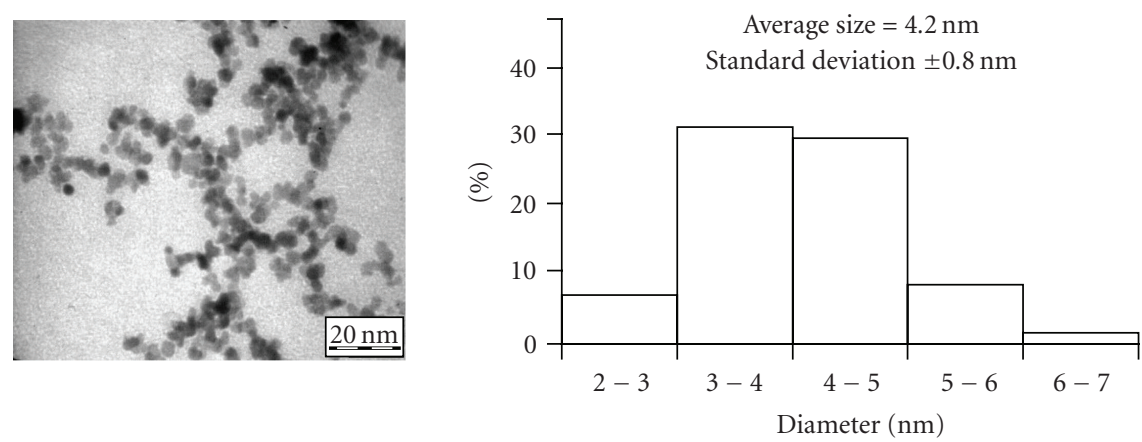

(d)

FIGURE 4: TEM micrographs, average size, standard deviation (SD), and size distribution of ENR-stabilized gold nanoparticles at (a) before and (b) after phase transfer (scale bar $=50 \mathrm{~nm}$ ) and platinum nanoparticles at (c) before and (d) after phase transfer $(\mathrm{scale}$ bar $=20 \mathrm{~nm})$. 
organic solvents that are compatible to ENR such as chloroform, tetrahydrofuran (THF), and the likes.

\section{ACKNOWLEDGMENT}

The authors acknowledge the grant awarded by Universiti Sains Malaysia (FRGS 203/PKIMIA/670049) in support of this work.

\section{REFERENCES}

[1] B. D. Summ and N. I. Ivanova, "The use of objects and methods of colloid chemistry in nanochemistry," Russian Chemical Reviews, vol. 69, no. 11, pp. 911-923, 2000.

[2] X. Zhong, R. Yuan, Y. Chai, Y. Liu, J. Dai, and D. Tang, "Glucose biosensor based on self-assembled gold nanoparticles and double-layer 2d-network (3-mercaptopropyl)trimethoxysilane polymer onto gold substrate," Sensors and Actuators B, vol. 104, no. 2, pp. 191-198, 2005.

[3] V. I. Bukhtiyarov and M. G. Slin'ko, "Metallic nanosystems in catalysis," Russian Chemical Reviews, vol. 70, no. 2, pp. 147159, 2001.

[4] M. Seydack, "Nanoparticle labels in immunosensing using optical detection methods," Biosensors and Bioelectronics, vol. 20, no. 12, pp. 2454-2469, 2005.

[5] C. Zhan, D. Li, D. Zhang, W. Xu, Y. Nie, and D. Zhu, "The excited-state absorption and third-order optical nonlinearity from 1-dodecanethiol protected gold nanoparticles: application for optical limiting," Optical Materials, vol. 26, no. 1, pp. $11-15,2004$

[6] H. X. He, H. Zhang, Q. G. Li, T. Zhu, S. F. Y. Li, and Z. F. Liu, "Fabrication of design architectures of Au nanoparticles on solid substrate with printed self-assembled monolayers as templates," Langmuir, vol. 16, no. 8, pp. 3846-3851, 2000.

[7] J. H. Song, Y.-J. Kim, and J.-S. Kim, "Synthesis of gold nanoparticles using N,N-dimethylacetoacetamide: size and shape control by the reaction temperature," Current Applied Physics, vol. 6, no. 2, pp. 216-218, 2006.

[8] N. Shirtcliffe, U. Nickel, and S. Schneider, "Reproducible preparation of silver sols with small particle size using borohydride reduction: for use as nuclei for preparation of larger particles," Journal of Colloid and Interface Science, vol. 211, no. 1, pp. 122-129, 1999.

[9] R. A. Caruso, M. Ashokkumar, and F. Grieser, "Sonochemical formation of gold sols," Langmuir, vol. 18, no. 21, pp. 78317836, 2002.

[10] W. Chen, J.-Y. Zhang, Y. Di, and I. W. Boyd, "Photo-chemical production of gold nanoparticles in monolithic porous silica by using a novel excimer ultraviolet source," Inorganic Chemistry Communications, vol. 6, no. 7, pp. 950-952, 2003.

[11] Y. Zhang, V. Suryanarayanan, I. Nakazawa, S. Yoshihara, and T. Shirakashi, "Electrochemical behavior of Au nanoparticle deposited on as-grown and O-terminated diamond electrodes for oxygen reduction in alkaline solution," Electrochimica Acta, vol. 49, no. 28, pp. 5235-5240, 2004.

[12] M. Brust, M. Walker, D. Bethell, D. J. Schiffrin, and R. Whyman, "Synthesis of thiol-derivatised gold nanoparticles in a two-phase liquid-liquid system," Journal of the Chemical Society, Chemical Communications, no. 7, pp. 801-802, 1994.

[13] S.-Y. Zhao, S.-H. Chen, D.-G. Li, X.-G. Yang, and H.-Y. Ma, "A convenient phase transfer route for Ag nanoparticles," Physica E, vol. 23, no. 1-2, pp. 92-96, 2004.
[14] K. V. Sarathy, G. U. Kulkarni, and C. N. R. Rao, "A novel method of preparing thiol-derivatised nanoparticles of gold, platinum and silver forming superstructures," Chemical Communications, no. 6, pp. 537-538, 1997.

[15] J. Yang, J. Y. Lee, T. C. Deivaraj, and H.-P. Too, "A highly efficient phase transfer method for preparing alkylaminestabilized Ru, Pt, and Au nanoparticles," Journal of Colloid and Interface Science, vol. 277, no. 1, pp. 95-99, 2004.

[16] A. Kumar, H. Joshi, R. Pasricha, A. B. Mandale, and M. Sastry, "Phase transfer of silver nanoparticles from aqueous to organic solutions using fatty amine molecules," Journal of Colloid and Interface Science, vol. 264, no. 2, pp. 396-401, 2003.

[17] C. Tian, Z. Kang, E. Wang, L. Gao, C. Wang, L. Xu, and C. Hu, "Synthesis of dodecanethiolate-protected $\mathrm{Cu}_{2} \mathrm{~S}$ nanoparticles in a two-phase system," Materials Letters, vol. 59, no. 10, pp. 1156-1160, 2005.

[18] S. Devarajan, B. Vimalan, and S. Sampath, "Phase transfer of $\mathrm{Au}-\mathrm{Ag}$ alloy nanoparticles from aqueous medium to an organic solvent: effect of aging of surfactant on the formation of Ag-rich alloy compositions," Journal of Colloid and Interface Science, vol. 278, no. 1, pp. 126-132, 2004.

[19] K. Esumi, T. Hosoya, A. Suzuki, and K. Torigoe, "Preparation of hydrophobically modified poly(amidoamine) dendrimerencapsulated gold nanoparticles in organic solvents," Journal of Colloid and Interface Science, vol. 229, no. 1, pp. 303-306, 2000.

[20] M. Abu Bakar, W. L. Tan, N. J. Azizi, and N. H. H. Abu Bakar, "Synthesis of modified natural rubber-stabilized silver organosols via liquid-to-liquid transfer techniques," Journal of Rubber Research, vol. 9, pp. 193-203, 2006.

[21] H. K. Lee, J. Ismail, H. W. Kammer, and M. A. Bakar, "Melt reaction in blends of poly(3-hydroxybutyrate) (PHB) and epoxidized natural rubber (ENR-50)," Journal of Applied Polymer Science, vol. 95, no. 1, pp. 113-129, 2005.

[22] J. Turkevich, P. C. Stevenson, and J. Hillier, "A study of the nucleation and growth processes in the synthesis of colloidal gold," Discussions of the Faraday Society, vol. 11, pp. 55-75, 1951.

[23] H. H. Ingelsten, R. Bagwe, A. Palmqvist, et al., "Kinetics of the formation of nano-sized platinum particles in water-inoil microemulsions," Journal of Colloid and Interface Science, vol. 241, no. 1, pp. 104-111, 2001.

[24] A. J. P. Van Zyl, S. M. Graef, R. D. Sanderson, B. Klumperman, and H. Pasch, "Monitoring the grafting of epoxidized natural rubber by size-exclusion chromatography coupled to FTIR spectroscopy," Journal of Applied Polymer Science, vol. 88, no. 10, pp. 2539-2549, 2003.

[25] K. L. Kelly, E. Coronado, L. L. Zhao, and G. C. Schatz, "The optical properties of metal nanoparticles: the influence of size, shape, and dielectric environment," Journal of Physical Chemistry B, vol. 107, no. 3, pp. 668-677, 2003.

[26] R. B. King, Ed., Encyclopedia of Inorganic Chemistry, Vol. 6, John Willey \& Sons, New York, NY, USA, 1994.

[27] K. B. Stavens, S. V. Pusztay, S. Zou, R. P. Andres, and A. Wei, "Encapsulation of neutral gold nanoclusters by resorcinarenes," Langmuir, vol. 15, no. 24, pp. 8337-8339, 1999.

[28] T. Kinoshita, S. Seino, K. Okitsu, T. Nakayama, T. Nakagawa, and T. A. Yamamoto, "Magnetic evaluation of nanostructure of gold-iron composite particles synthesized by a reverse micelle method," Journal of Alloys and Compounds, vol. 359, no. 1-2, pp. 46-50, 2003.

[29] J. Shim, D.-Y. Yoo, and J.-S. Lee, "Characteristics for electrocatalytic properties and hydrogen-oxygen adsorption of 
platinum ternary alloy catalysts in polymer electrolyte fuel cell," Electrochimica Acta, vol. 45, no. 12, pp. 1943-1951, 2000.

[30] D.-G. Li, S.-H. Chen, S.-Y. Zhao, X.-M. Hou, H.-Y. Ma, and X.G. Yang, "A study of phase transfer processes of Ag nanoparticles," Applied Surface Science, vol. 200, no. 1-4, pp. 62-67, 2002.

[31] H. Zhu, C. Tao, S. Zheng, S. Wu, and J. Li, "Effect of alkyl chain length on phase transfer of surfactant capped Au nanoparticles across the water/toluene interface," Colloids and Surfaces A, vol. 256, no. 1, pp. 17-20, 2005.

[32] J. Yang, J. Y. Lee, T. C. Deivaraj, and H. P. Too, "An improved Brust's procedure for preparing alkylamine stabilized $\mathrm{Pt}, \mathrm{Ru}$ nanoparticles," Colloids and Surfaces A, vol. 240, no. 1-3, pp. 131-134, 2004.

[33] M. Sastry, A. Kumar, and P. Mukherjee, "Phase transfer of aqueous colloidal gold particles into organic solutions containing fatty amine molecules," Colloids and Surfaces A, vol. 181, no. 1-3, pp. 255-259, 2001.

[34] S. Gubin, "Metalcontaining nano-particles within polymeric matrices: preparation, structure, and properties," Colloids and Surfaces A, vol. 202, no. 2-3, pp. 155-163, 2002. 

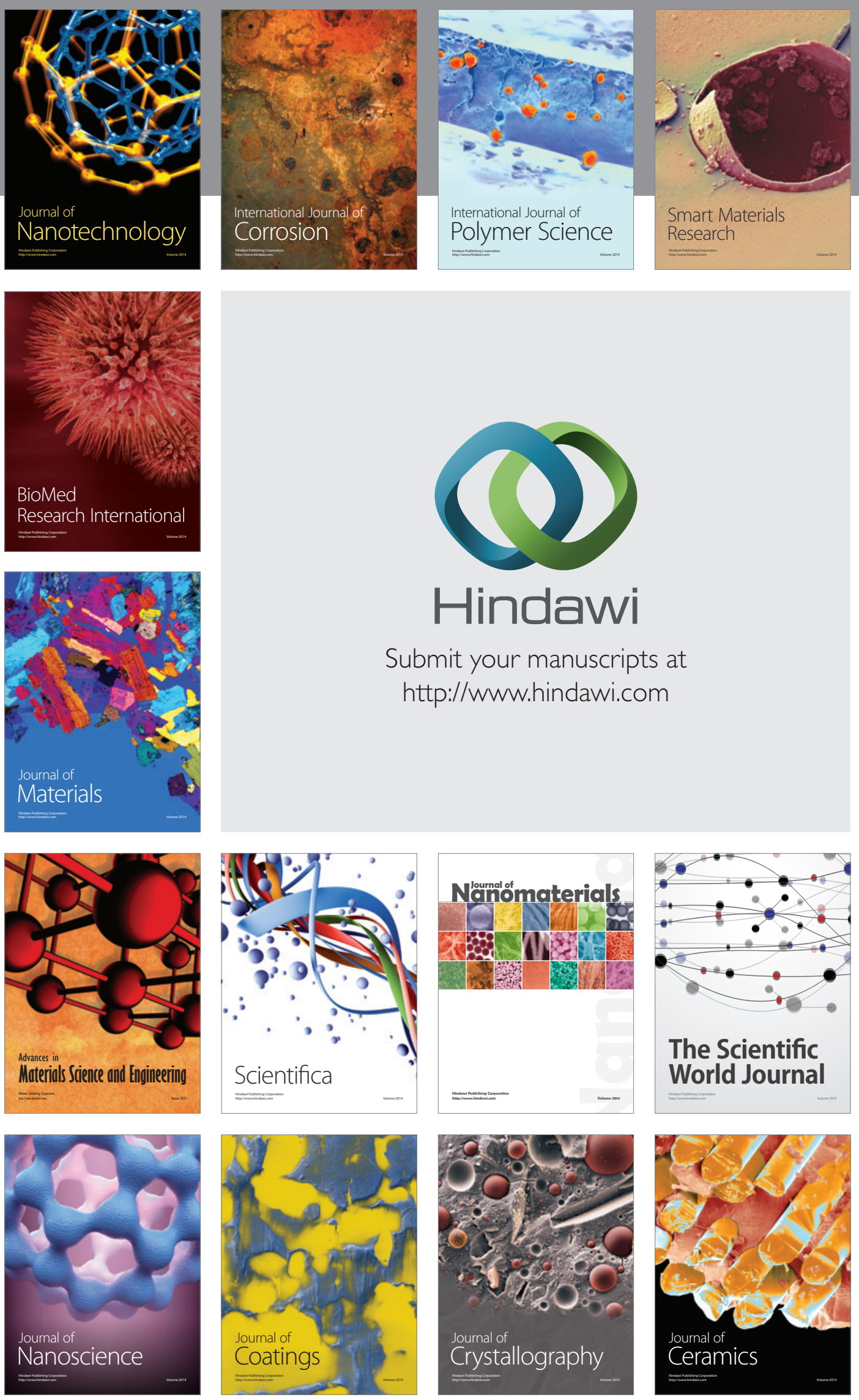

The Scientific World Journal

Submit your manuscripts at

http://www.hindawi.com

\section{World Journal}

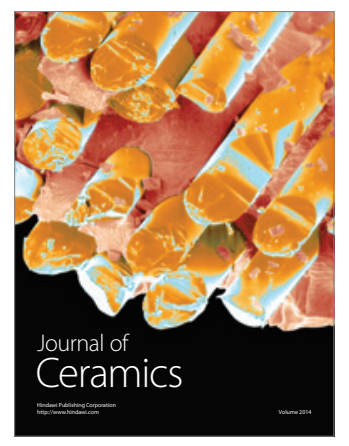

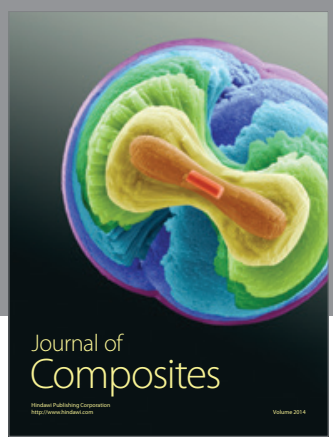
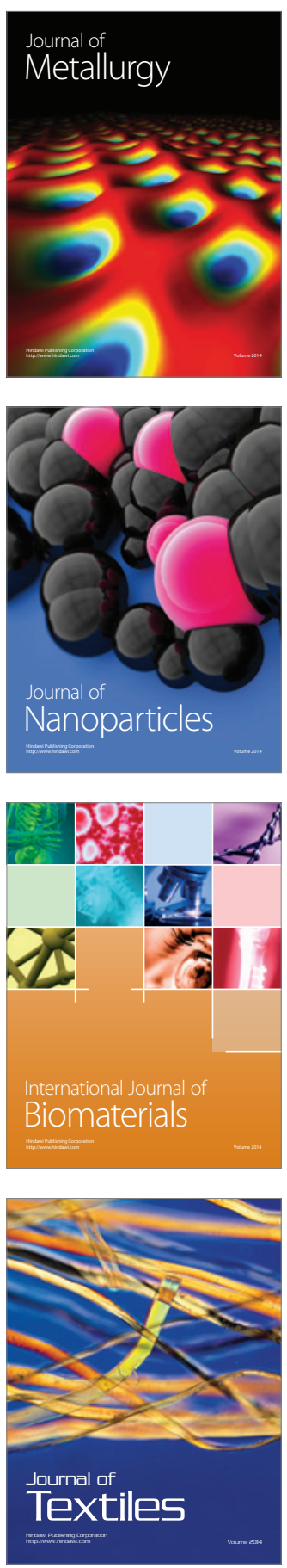